\title{
Standard versus bicaval techniques for orthotopic heart \\ transplantation: An analysis of the United Network for Organ Sharing database
}

\author{
Ryan R. Davies, MD, ${ }^{\text {a }}$ Mark J. Russo, MD, MS, ${ }^{\mathrm{b}}$ Jeffrey A. Morgan, MD, ${ }^{\mathrm{a}}$ Robert A. Sorabella, BA, ${ }^{\mathrm{a}}$ \\ Yoshifumi Naka, MD, PhD, ${ }^{\mathrm{a}}$ and Jonathan M. Chen, $\mathrm{MD}^{\mathrm{a}}$
}

\begin{abstract}
Objective: Most studies of anastomotic technique have been underpowered to detect subtle differences in survival. We analyzed the United Network for Organ Sharing database for trends in use and outcomes after either bicaval or traditional (biatrial) anastomoses for heart implantation.
\end{abstract}

\begin{abstract}
Methods: Review of United Network for Organ Sharing data identified 20,999 recipients of heart transplants from 1997 to 2007. Patients were stratified based on the technique of atrial anastomosis: standard biatrial (atrial group, $\mathrm{n}=11,919,59.3 \%$ ), bicaval (caval group, $\mathrm{n}=7661,38.1 \%$ ), or total orthotopic (total group, $\mathrm{n}=519$, $2.6 \%$ ).

Results: The use of the bicaval anastomosis is increasing, but many transplantations continue to use a biatrial anastomosis $(1997,0.2 \%$ vs $97.6 \% ; 2007,62.0 \%$ vs $34.7 \% ; P<.0001)$. Atrial group patients required permanent pacemaker implantation more often (odds ratio, 2.6; 95\% confidence interval, 2.2-3.1). Caval group patients had a significant advantage in 30-day mortality (odds ratio, 0.83; 95\% confidence interval, 0.75-0.93), and Cox regression analysis confirmed the decreased long-term survival in the atrial group (hazard ratio, $1.11 ; 95 \%$ confidence interval, 1.04-1.19).
\end{abstract}

Conclusions: Heart transplantations performed with bicaval anastomoses require postoperative permanent pacemaker implantation at lower frequency and have a small but significant survival advantage compared with biatrial anastomoses. We recommend that except where technical considerations require a biatrial technique, bicaval anastomoses should be performed for heart transplantation. (J Thorac Cardiovasc Surg 2010;140:700-8)

\section{Supplemental material is available online.}

\footnotetext{
After a fitful start in the late $1960 \mathrm{~s},{ }^{1}$ cardiac transplantation has become the treatment of choice for end-stage heart failure. Over the ensuing 40 years, significant advances have been made in both the perioperative management of donors and recipients and in long-term management to prevent and

From the Division of Cardiothoracic Surgery ${ }^{a}$ and the International Center for Health Outcomes and Innovation Research, ${ }^{b}$ Department of Surgery, Columbia University College of Physicians and Surgeons, and the Department of Surgery, Weill Medical College of Cornell University, New York, NY.

Supported in part by Health Resources and Services Administration contract 231-000115 and departmental funding sources. The content of this article is the responsibility of the authors alone and does not necessarily reflect the views or policies of the Department of Health and Human Services, nor does mention of trade names, commercial products, or organizations imply endorsement by the US Government

Read at the Annual Meeting of the International Society for Heart \& Lung Transplantation, Boston, Mass, April 2008.

Received for publication March 8, 2009; revisions received Jan 12, 2010; accepted for publication April 26, 2010; available ahead of print June 28, 2010.

Address for reprints: Jonathan M. Chen, MD, Pediatric Cardiac Surgery Children's Hospital of New York, 3959 Broadway, Suite 2-273, New York, NY 10032

(E-mail: jmc23@columbia.edu).

0022-5223/\$36.00

Copyright (c) 2010 by The American Association for Thoracic Surgery doi:10.1016/j.jtcvs.2010.04.029
}

treat rejection; however, the most commonly performed technique of cardiac allograft implantation, the standard, or biatrial, technique, has remained little changed since its initial description by Lower and Shumway in $1960 .^{2}$

The primary advantage of the biatrial technique is its relative simplicity. By performing only 2 anastomoses to the donor atria (rather than 6 to the 2 cavae and the 4 pulmonary veins), the technical challenge of cardiac implantation is lessened, and allograft ischemic times are reduced. However, the biatrial anastomosis puts the sinoatrial node at risk of injury, and redundant atrial tissue might worsen atrial hemodynamics and contribute to an increased risk of atrial arrhythmias in the postoperative period. ${ }^{3,4}$

Attempts to solve these downsides resulted in the development of 2 alternative techniques: bicaval and total heterotopic cardiac transplantation. ${ }^{5-7}$ The bicaval anastomosis consists of a single left atrial anastomosis with separate caval suture lines, whereas total heterotopic transplantation divides the left atrial anastomosis into 2 portions (left and right pulmonary veins). These techniques are progressively more complicated and might require longer operative times, but they should result in a lower incidence of sinoatrial node dysfunction and improved hemodynamic and physiologic cardiac performance. Unfortunately, studies performed to date to assess the results of these techniques have generally been insufficiently powered (because of either small sample 


\section{Abbreviations and Acronyms \\ $\mathrm{CI}=$ confidence interval \\ $\mathrm{PPM}=$ permanent pacemaker \\ UNOS $=$ United Network for Organ Sharing}

sizes $^{8-14}$ or high frequency of missing data ${ }^{15}$ ) to detect important differences.

This report uses data from the United Network for Organ Sharing (UNOS) database to assess posttransplantation outcomes in patients stratified by the technique used for allograft implantation. Our goals were (1) to assess the current use of these techniques and (2) to identify which techniques resulted in better short- and long-term outcomes.

\section{MATERIALS AND METHODS \\ Data Collection}

UNOS provided deidentified patient-level, encrypted center-specific data from the Thoracic Registry (data source no. 092707-15). The UNOS Standard Transplant and Research Dataset contains information from the UNOS forms, including the Transplant Candidate Registration form, the Transplant Recipient Registration form, and the Transplant Recipient Follow-up form. Use of these data is consistent with the regulations of our university's institutional review board.

\section{Study Population}

The study population consisted of 20,999 transplantations performed on adult patients without congenital heart disease between 1997 and 2007 for whom UNOS data included the type of anastomosis performed. Patients were stratified based on the technique of atrial anastomosis: standard biatrial (atrial group, $\mathrm{n}=11,919$ [59.3\%]), bicaval (caval group, $\mathrm{n}=7661$ $[38.1 \%]$ ), or total orthotopic (total group, $n=519[2.6 \%]$ )

\section{Data Analysis}

Statistical analysis was performed with SAS 9.13 software for Windows (SAS Institute, Inc, Cary, NC). Primary outcomes were (1) the need for a permanent pacemaker (PPM) before hospital discharge after transplantation and (2) 30-day mortality. Other outcomes included length of stay, the incidence of in-hospital complications, long-term survival, graft survival, transplant coronary artery disease-free survival, and renal failure-free survival. Continuous variables are reported as means \pm standard deviations and were compared by using the Student's $t$ test.

Ordinal variables were compared by using the $\chi^{2}$ test. Body surface area, weight, and age were used as both continuous variables and stratified into subgroups; the most predictive method was used. $P$ values are all 2 -sided. Risk ratios and $95 \%$ confidence intervals (CIs) are also reported. KaplanMeier analysis and Cox proportional hazards regression (PROC PHREG, stepwise, $P<.05$ ) were used for time-to-event analysis. Survival function estimates for strata of the explanatory variables were calculated with the BASELINE statement of PROC PHREG. Multivariate regression (PROC LOGISTIC, stepwise, $P<.05$ ) was also performed to evaluate the incidence of peritransplantation death and the incidence of morbidity; all variables reaching statistical significance in univariate analysis were included in multivariate analyses.

\section{Missing Variables}

Missing variables are a significant problem in analyses of large multiinstitutional databases in general and the UNOS database in particular (see Table 1 for the proportion of missing data for each variable in our dataset). For multivariate analyses, missing variables were imputed by using the technique of multiple imputation, as implemented by using the MI and MIANALYZE procedures (SAS 9.13 for Windows; see the Materials and Methods section of this article's Online Repository for full details of analysis). Multiple imputation has been shown to produce efficient and unbiased estimates if the data are missing at random and even in the rarer situation of information missing not at random. ${ }^{16-19}$ In circumstances with a high proportion of missing variables, as is the case with the UNOS dataset (see again), it provides more accurate estimates of true relationships than the more commonly used method of listwise deletion. A full list of evaluated variables is given in Table E2 in the Materials and Methods section of this article's Online Repository.

The authors had full access to the data and take full responsibility for its integrity. All authors have read and agree with the manuscript as written.

\section{RESULTS}

\section{Anastomotic Technique}

Baseline demographics and clinical status at the time of transplantation in patients undergoing each anastomotic technique are shown in Table 2. Since 1997, the use of bicaval techniques has been steadily increasing, whereas the number of total orthotopic transplantations has been decreasing $(P=.0001$, Figure 1). However, 644 (34.5\%) transplantations in 2006 were still performed with a biatrial anastomosis. The percentage of transplantations performed with the bicaval technique was higher at higher-volume transplant centers $(P=.0344$, Figure 2$)$.

\section{Short-Term Outcomes}

Patients in the atrial group $(n=576,5.1 \%)$ required a PPM before discharge more often (odds ratio [vs the caval group], 2.6; 95\% CI, 2.2-3.1) than those in the caval group $(\mathrm{n}=146,2.0 \%)$ or the total group $(\mathrm{n}=11,1.9 \%$; odds ratio [vs the caval group], 1.0, 95\% CI, 0.6-1.7). Multivariate predictors of the need for PPM implantation included biatrial anastomosis (odds ratio, 3.1; 95\% CI, 2.5-3.9), donor age of 60 to 69 years (odds ratio, 2.9; 95\% CI, 1.5-5.3), donor age of 50 to 59 years (odds ratio, 2.0; 95\% CI, 1.6-2.5), donor age of 40 to 49 years (odds ratio, 1.3 ; $95 \%$ CI, 1.0-1.6), recipient inotropic support at transplantation (odds ratio, 1.5; 95\% CI, 1.2-1.7), donor history of hypertension (odds ratio, $1.2 ; 95 \% \mathrm{CI}, 1.0-1.4$ ), and transplantation year (odds ratio, 1.04; 95\% CI 1.01-1.07 [per year]); use of T4 before organ retrieval (odds ratio, $0.8 ; 95 \% \mathrm{CI}, 0.6-0.9$ ) was protective. Length of stay was similar between patients requiring and not requiring PPM implantation (21.8 vs 20.0 days, $P=.1360)$. Despite this, patients in the atrial group had longer posttransplantation lengths of-stay (21.1 days) than those in the caval group (19.3 days, $P<.0001)$.

In univariate analysis atrial group patients had a higher incidence of postoperative death $(8.9 \%$; odds ratio, $1.17 ; 95 \%$ CI, 1.05-1.30) than those in the caval group (7.6\%; odds ratio, $0.83 ; 95 \%$ CI, $0.75-0.93$ ); postoperative mortality in the total group (9.5\%; odds ratio, $1.14 ; 95 \% \mathrm{CI}, 0.86-1.53$ ) was not significantly different from that seen in either of the other 
TABLE 1. Frequency of missing data for all variables included in multivariate models predicting both long-term and short-term risk of mortality $(n=20,069) *$

\begin{tabular}{|c|c|}
\hline Risk factor & Percentage missing \\
\hline Albumin level at listing & 43.5 \\
\hline Previous sternotomy & 35.2 \\
\hline Panel reactive antibody level & 35.0 \\
\hline Donor on $\mathrm{T} 3$ at organ retrieval & 30.3 \\
\hline History of hepatitis B & 12.0 \\
\hline Organ ischemic time & 9.3 \\
\hline Transfusion between listing and transplantation & 9.2 \\
\hline Bilirubin level at transplantation & 7.8 \\
\hline Donor on T4 at organ retrieval & 7.7 \\
\hline History of COPD & 7.3 \\
\hline Creatinine clearance at transplantation & 5.6 \\
\hline BMI at transplantation & 5.6 \\
\hline Dialysis at transplantation & 4.2 \\
\hline Recipient/donor weight ratio & 3.6 \\
\hline History of stroke & 3.0 \\
\hline History of hepatitis C & 2.2 \\
\hline Donor bilirubin & 2.2 \\
\hline History of diabetes & 1.9 \\
\hline Donor history of hypertension & 0.7 \\
\hline Donor cause of death & 0.2 \\
\hline Donor age & 0.1 \\
\hline Intravenous antibiotics within $2 \mathrm{wk}$ & 0.06 \\
\hline Hospital location (ICU vs non-ICU vs out of hospital) & 0.01 \\
\hline Status at transplantation & 0.01 \\
\hline Cause & 0.0 \\
\hline Sex & 0.0 \\
\hline Use of extracorporeal membrane oxygenation & 0.0 \\
\hline Use of ventricular assist device & 0.0 \\
\hline Use of intra-aortic balloon pump & 0.0 \\
\hline Mechanical ventilation & 0.0 \\
\hline Inhaled nitric oxide & 0.0 \\
\hline Prostaglandin E & 0.0 \\
\hline Donor ABO type & 0.0 \\
\hline Donor cigarette use & 0.0 \\
\hline Donor sex & 0.0 \\
\hline Donor on inotropes/pressors & 0.0 \\
\hline Recipient $\mathrm{ABO}$ type & 0.0 \\
\hline Transplantation year & 0.0 \\
\hline Anastomosis: total & $0.0 \% \dagger$ \\
\hline Anastomosis: biatrial & $0.0 \% \dagger$ \\
\hline
\end{tabular}

$C O P D$, Chronic obstructive pulmonary disease; $B M I$, body mass index; $I C U$, intensive care unit. *Variables with zero missing values are often variables calculated based on responses where no option was given for "no," and thus null results are assumed to be "no" responses, resulting in no missing values. †Because type of anastomosis was the focus of this analysis, missing values were not imputed for this variable, and $327 \mathrm{pa}-$ tients without anastomotic data were excluded.

groups. However, the logistic regression model predicting postoperative death did not include type of anastomosis (Table 3).

\section{Long-Term Outcomes}

The long-term hazard of PPM implantation was also significantly higher among patients in the atrial group
$(P<.0001$, Figure 3$)$; at 2 years, $8.6 \%$ required a pacemaker versus only $5.4 \%$ in the caval group and $4.0 \%$ in the total group. Multivariate predictors of time to posttransplantation PPM insertion included recipient age (odds ratio, 1.006; 95\% CI, 1.001-1.012 [per year]), transfusions between listing and transplantation (odds ratio, 1.2; 95\% CI, 1.0-1.4), donor age of 50 to 59 years (odds ratio, 1.6; 95\% CI, 1.3-2.0), donor age of 60 to 69 years (odds ratio, 2.2; 95\% CI, 1.3-3.7), transplantation year (odds ratio, 1.25; 95\% CI, 1.21-1.28 [per year]), and biatrial anastomosis (odds ratio, 2.5; 95\% CI, 2.2-2.9); ventricular assist device at transplantation was protective in this model (odds ratio, $0.7 ; 95 \%$ CI, 0.6-0.9).

There was a small but significant difference in long-term survival between the atrial and caval groups in univariate analysis (survival at 1 year, $85.6 \%$ vs $87.1 \%$; at 5 years, $72.2 \%$ vs $73.5 \%$; at 10 years, $51.1 \%$ vs $57.4 \%$; $P<.0168$; Figure 4). Multivariate Cox proportional hazards regression analysis confirmed the decreased survival among patients in the atrial group (hazard ratio, $1.11 ; 95 \% \mathrm{CI}$, 1.04-1.19; Table 4). There was no difference in graft survival, renal failure-free survival, and transplant coronary atherosclerosis-free survival based on anastomotic technique.

\section{DISCUSSION}

Our results demonstrate a small but significant survival advantage in multivariate analysis for patients undergoing a bicaval anastomosis for cardiac allograft implantation. Heretofore, the evidence in the literature has been somewhat contradictory. Although some previous studies demonstrated a survival advantage with the use of the bicaval technique, ${ }^{20-22}$ which was confirmed in a meta-analysis by Schnoor and colleagues, ${ }^{8}$ a recent analysis of the UNOS dataset by Weiss and associates ${ }^{15}$ showed no difference between groups.

The smaller studies ${ }^{20-22}$ (and by extension the metaanalysis ${ }^{8}$ ) are all susceptible to bias because they used only univariate analysis and failed to account for the correlation between the use of the bicaval technique and increased experience over time. However, an analysis of the UNOS dataset from 1999 to 2005 by Weiss and associates ${ }^{15}$ did not show a similar advantage. We hope that our results might clarify the reasons for this discrepancy in the literature and confirm the advantages of the bicaval technique.

Although we have analyzed a similar patient population, our results appear to conflict with the recently published analysis by Weiss and associates, ${ }^{15}$ also looking at the UNOS dataset. The easy justification for the different results obtained here would be the additional patients we were able to include by using data through 2007; however, we speculate that more interesting and more important differences in analytic techniques explain the differing results. These 
TABLE 2. Pretransplantation demographic and clinical variables divided by type of transplant anastomosis

\begin{tabular}{|c|c|c|c|c|}
\hline & Atrial group & Caval group & Total group & $P$ value \\
\hline Sex (male) & $9054(75.9 \%)$ & $5815(77.0 \%)$ & $456(76.0 \%)$ & .23 \\
\hline Age (mean $\pm 95 \% \mathrm{CI})$ & $52.2 \pm 0.3$ & $51.9 \pm 0.3$ & $51.9 \pm 1.1$ & .0001 \\
\hline \multicolumn{5}{|l|}{ Cause of heart failure } \\
\hline Dilated cardiomyopathy & $9784(82.1 \%)$ & $6095(80.7 \%)$ & $502(83.7 \%)$ & .02 \\
\hline Congenital heart disease & $241(2.0 \%)$ & $204(2.7 \%)$ & $19(3.2 \%)$ & .003 \\
\hline Ischemic cardiomyopathy & $878(7.4 \%)$ & $556(7.4 \%)$ & $29(4.8 \%)$ & .06 \\
\hline Retransplantation & $329(2.8 \%)$ & $241(3.2 \%)$ & $18(3.0 \%)$ & .22 \\
\hline Hypertrophic cardiomyopathy & $173(1.5 \%)$ & $135(1.8 \%)$ & $5(0.8 \%)$ & .06 \\
\hline Restrictive cardiomyopathy & $181(1.5 \%)$ & $127(1.7 \%)$ & $8(1.3 \%)$ & .60 \\
\hline \multicolumn{5}{|l|}{ ABO blood type } \\
\hline A & $5146(43.2 \%)$ & $3240(42.9 \%)$ & $250(41.7 \%)$ & .74 \\
\hline $\mathrm{B}$ & $1601(13.4 \%)$ & $1033(13.7 \%)$ & $81(13.5 \%)$ & .88 \\
\hline $\mathrm{AB}$ & $604(5.1 \%)$ & $385(5.1 \%)$ & $31(5.2 \%)$ & .99 \\
\hline $\mathrm{O}$ & $4568(38.3 \%)$ & $2892(38.3 \%)$ & $238(39.7 \%)$ & .80 \\
\hline Previous sternotomy & $2981(50.9 \%)$ & $3129(47.2 \%)$ & $274(53.1 \%)$ & .0001 \\
\hline Days on waiting list (mean $\pm 95 \% \mathrm{CI}$ ) & $241 \pm 8$ & $196 \pm 10$ & $158 \pm 25$ & .0001 \\
\hline \multicolumn{5}{|l|}{ Laboratory values at transplantation } \\
\hline Creatinine clearance $<40 \mathrm{~mL} / \mathrm{min}$ & $536(4.8 \%)$ & $391(5.4 \%)$ & $31(5.3 \%)$ & .17 \\
\hline Total bilirubin $>2.0 \mathrm{mg} / \mathrm{dL}$ & $1207(11.1 \%)$ & $850(12.0 \%)$ & $69(12.5 \%)$ & .14 \\
\hline Albumin $<3.5 \mathrm{mg} / \mathrm{dL}$ & $1909(34.6 \%)$ & $1906(35.7 \%)$ & $169(35.8 \%)$ & .43 \\
\hline \multicolumn{5}{|l|}{ Medical history } \\
\hline Previous stroke & $726(6.3 \%)$ & $411(5.6 \%)$ & $42(7.1 \%)$ & .05 \\
\hline Diabetes & $2326(20.0 \%)$ & $1676(22.4)$ & $151(25.4 \%)$ & .0001 \\
\hline Chronic obstructive pulmonary disease & $375(3.4 \%)$ & $229(3.3 \%)$ & $31(5.5 \%)$ & .02 \\
\hline \multicolumn{5}{|l|}{ Clinical status } \\
\hline Status $1 / 1 \mathrm{~A} / \mathrm{IB}$ at transplantation & $8683(72.9 \%)$ & $5710(75.6 \%)$ & $465(77.5 \%)$ & .0001 \\
\hline Inotropes & $5744(48.2 \%)$ & $3663(48.5 \%)$ & $291(48.5 \%)$ & .90 \\
\hline Hospitalized (non-ICU) & $2002(16.8 \%)$ & $1587(21.0 \%)$ & $124(20.7 \%)$ & .0001 \\
\hline $\mathrm{ICU}$ & $4737(39.8 \%)$ & $2313(30.6 \%)$ & $240(40.0 \%)$ & .0001 \\
\hline Mechanical ventilation & $370(3.1 \%)$ & $200(2.7 \%)$ & $19(3.2 \%)$ & .18 \\
\hline Ventricular assist device & $2174(18.2 \%)$ & $1624(21.5 \%)$ & $118(19.7 \%)$ & .0001 \\
\hline Extracorporeal membrane oxygenation & $39(0.3 \%)$ & $33(0.4 \%)$ & $1(0.2 \%)$ & .33 \\
\hline Transfusion between listing and transplantation & $2129(19.9 \%)$ & $1484(21.4 \%)$ & $82(14.3 \%)$ & .0001 \\
\hline Antibiotics within $2 \mathrm{wk}$ of transplantation & $1208(10.1 \%)$ & $834(11.1 \%)$ & $78(13.0 \%)$ & .0189 \\
\hline Intra-aortic balloon pump & $591(5.0 \%)$ & $434(5.8 \%)$ & $44(7.3 \%)$ & .0049 \\
\hline Transplantation year (mean) & 2000 & 2003 & 2002 & .0001 \\
\hline
\end{tabular}

$C I$, Confidence interval; $I C U$, intensive care unit.

differences, revolving around the handling of missing data, merit detailed discussion because they influence any analysis of the UNOS dataset.

A complete discussion of the statistical analysis of datasets containing missing data is well beyond the scope of this article; however, because of its importance to this data analysis, we will briefly address the advantages of multiple imputation over other methods of dealing with missing data, including listwise deletion (also known as complete case analysis) and simple imputation. The appropriate handling of missing data is essential to any analysis of the UNOS dataset because it contains a significant amount of missing data (see Table E1 and the Materials and Methods section of this article's Online Repository).

As with most analyses of large datasets (in part because it is the default setting of statistical programs), Weiss and as- sociates ${ }^{15}$ have used complete case analysis, in which cases missing variables included in the multivariate model are eliminated from the analysis. This technique, although common, ${ }^{23}$ assumes that data are missing completely at random (for a more complete discussion of the types of missing data, see the Materials and Methods section of this article's Online Repository); that is, it assumes that the group of patients with complete data is identical to the entire group of interest (in this case all adult patients undergoing transplantation). This assumption is rarely borne out in analyses of missing data in medical datasets, and despite its wide application, the use of complete case analysis often leads to substantial bias and poor predictive value. ${ }^{17}$

Simple imputation is another commonly used method to account for missing values in large datasets. This involves the replacement of all missing values for a particular variable 


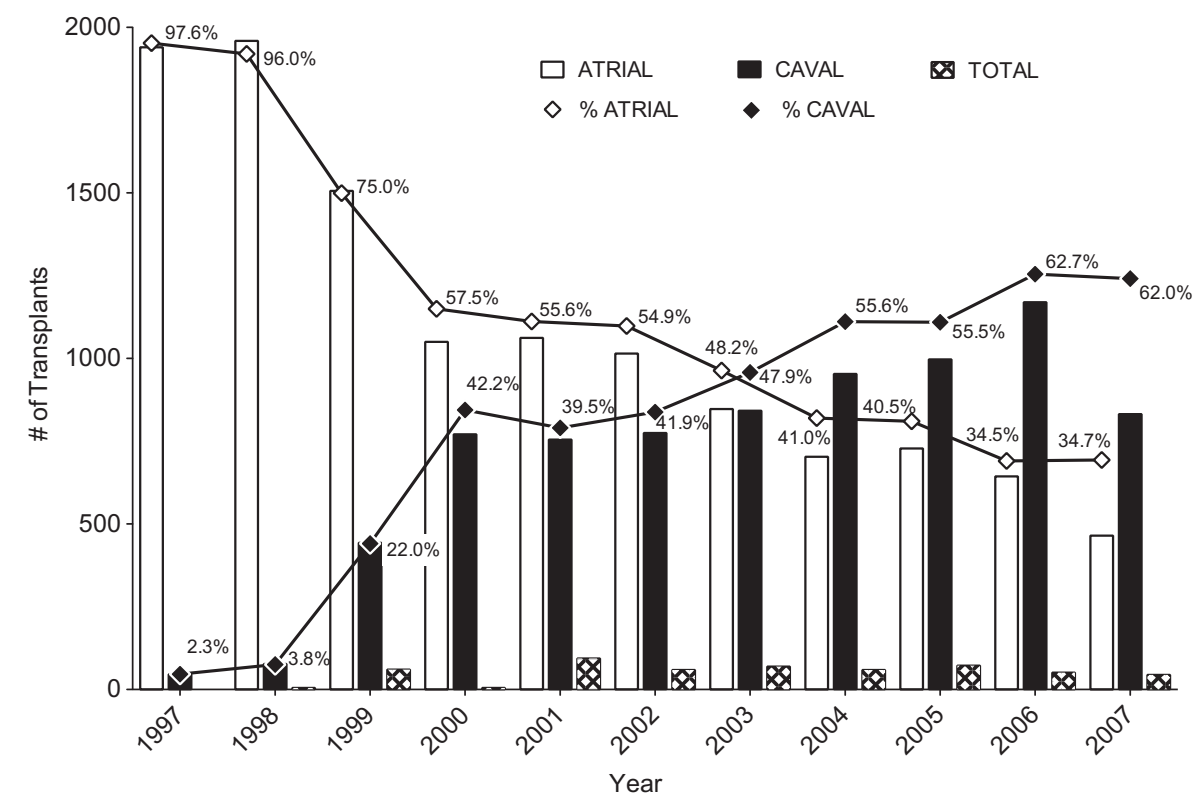

FIGURE 1. Transplantations performed by using each anastomosis type, as reported to the UNOS database by year from 1997 to 2007 . White columns, Number of biatrial anastomoses; black columns, number of bicaval anastomoses; cross-hatched columns, number of total orthotopic anastomosis. Lines indicate the percentage of transplantations in that year performed with either biatrial (white diamonds) or bicaval (black diamonds) anastomosis. $P<.0001$.

with a particular result (often either the mean or mode over the whole population). Although simple to implement and easy to understand, it results in significant bias unless the missing values are missing completely at random (ie, the probability of a particular observation being missing is not dependent on the value of that observation or any other variable in the dataset).

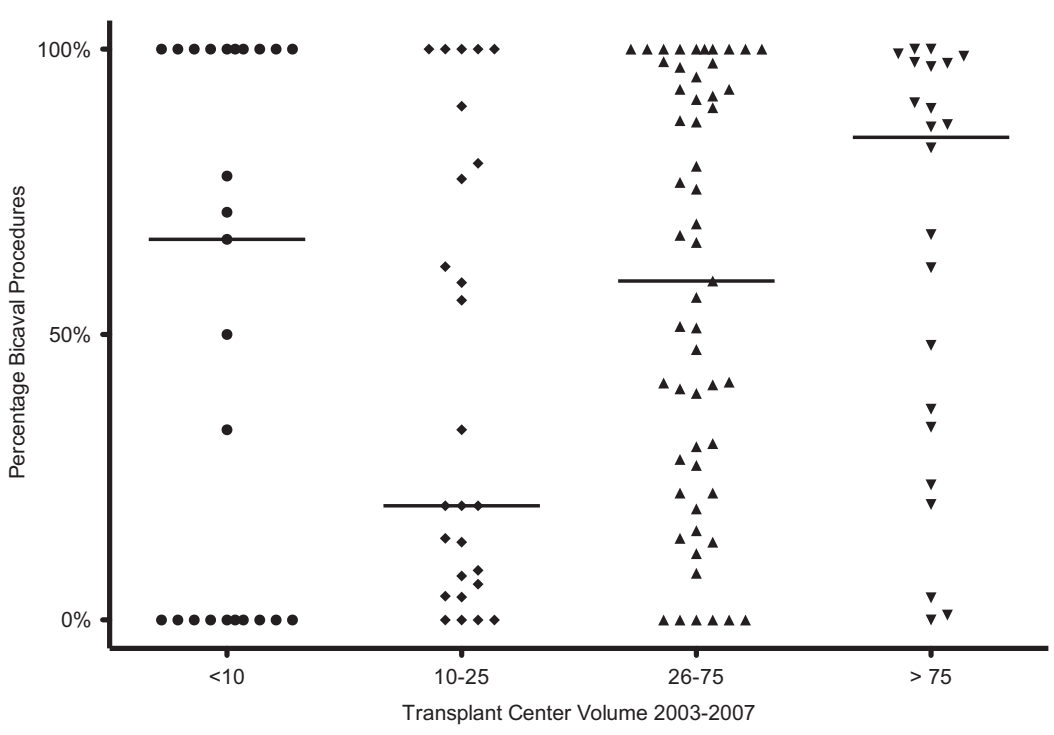

FIGURE 2. Scatter graph illustrating correlation between increasing transplantations and a larger percentage of transplantations performed with a bicaval anastomosis. Patients were divided into 4 groups based on total volume of transplantations performed from 2003 to 2007: (<10 [circles], 10-25 [diamonds], 26-75 [up-pointing triangles], and $>75$ [down-pointing squares]). Each marker represents a single transplant center and is plotted on the $x$-axis by the transplant center volume and on the $y$-axis by the percentage of transplantations performed by using bicaval anastomosis. Horizontal line indicates median percentage of bicaval transplantations performed in each volume group. $P=.0344$ for correlation between increasing volume and increasing percentage of bicaval transplantations.
We have chosen to use the technique of multiple imputation. Although this technique was developed to account for data that were missing at random (ie, where the value of a particular variable does not influence the probability of it being missing), it has been demonstrated to provide accurate approximations with appropriate $95 \%$ CIs, even where data is missing not at random (see the Materials and Methods section 
TABLE 3. Multivariate logistic regression model of posttransplantation death (within 30 days or before discharge) among patients with reported transplant anastomosis

\begin{tabular}{lcc}
\hline \multicolumn{1}{c}{ Risk factor } & Odds ratio & $\mathbf{9 5} \%$ CI \\
\hline Extracorporeal membrane oxygenation & 5.00 & $2.43-10.30$ \\
Cause: congenital heart disease & 4.38 & $3.18-6.03$ \\
Prostaglandin E & 3.67 & $1.41-9.52$ \\
Mechanical ventilation & 2.85 & $2.24-3.63$ \\
Creatinine clearance $<40 \mathrm{~mL} / \mathrm{min}$ & 2.26 & $1.85-2.78$ \\
Bilirubin $>2.0 \mathrm{mg} / \mathrm{dL}$ & 1.95 & $1.66-2.30$ \\
Ventricular assist device & 1.87 & $1.60-2.19$ \\
Cause: retransplantation & 1.85 & $1.36-2.52$ \\
Donor age $50-59$ y & 1.71 & $1.38-2.10$ \\
Ischemic time $>4$ h & 1.64 & $1.42-1.89$ \\
Cause: restrictive cardiomyopathy & 1.47 & $0.94-2.31$ \\
Cause: hypertrophic cardiomyopathy & 1.46 & $0.89-2.38$ \\
Peak PRA $>40 \%$ & 1.38 & $1.10-1.74$ \\
Donor age 40-49 y & 1.36 & $1.14-1.61$ \\
Donor age $30-39$ y & 1.25 & $1.06-1.47$ \\
Intravenous antibiotics within 2 wk & 1.19 & $1.00-1.42$ \\
Donor cause of death: stroke & 1.17 & $1.01-1.35$ \\
Cause: ischemic cardiomyopathy & 1.14 & $0.92-1.42$ \\
Cause: valvular cardiomyopathy & 1.06 & $0.73-1.54$ \\
Age (risk per additional year) & 1.022 & $1.016-1.028$ \\
Transplantation year & 0.93 & $0.92-0.95$ \\
Sex (male) & 0.82 & $0.7-0.94$ \\
\hline PRA, Par & &
\end{tabular}

PRA, Panel reactive antibody.

of this article's Online Repository for more information). ${ }^{24,25}$ In addition, by including patients with some missing information, a much larger sample size can be used in the multivariate analysis, permitting the identification of less powerful but still significant predictors of outcomes. Thus multiple imputation allows more accurate inferences about the entire population of interest (rather than only the population with complete data), enabling both more precise and more powerful data analysis. Details of our method of multiple imputation are given in the Materials and Methods section of this article's Online Repository.

To return specifically to the analysis of transplant anastomosis provided in this article, we were able to identify biatrial anastomosis as having a hazard ratio for mortality in Cox proportional hazards regression of $1.11(95 \% \mathrm{CI}$, 1.04-1.19). This is in fact similar to the odds ratio shown by Weiss and associates (1.06), ${ }^{15}$ except that their CIs were wider and did not reach statistical significance. Even in their analysis, it was similar in magnitude (if not in statistical significance) to the hazard ratio for prolonged ischemic time. ${ }^{15}$ Therefore although they concluded that "there was no difference in survival between the bicaval and biatrial technique," we believe, and our analysis here supports, that this was a matter of insufficient power because of the exclusion of patients with missing data rather than the lack of effect.

Unfortunately, because of the limitations of the UNOS dataset, we can only speculate as to the cause of this difference in long-term mortality. Although previous studies have not been sufficiently powered to demonstrate differences in long-term mortality based on the anastomotic technique during transplantation by using multivariate techniques, several studies have demonstrated benefits to atrial physiology in the posttransplantation period. These include improved atrial emptying and decreased atrial diameter ${ }^{4,26}$ and

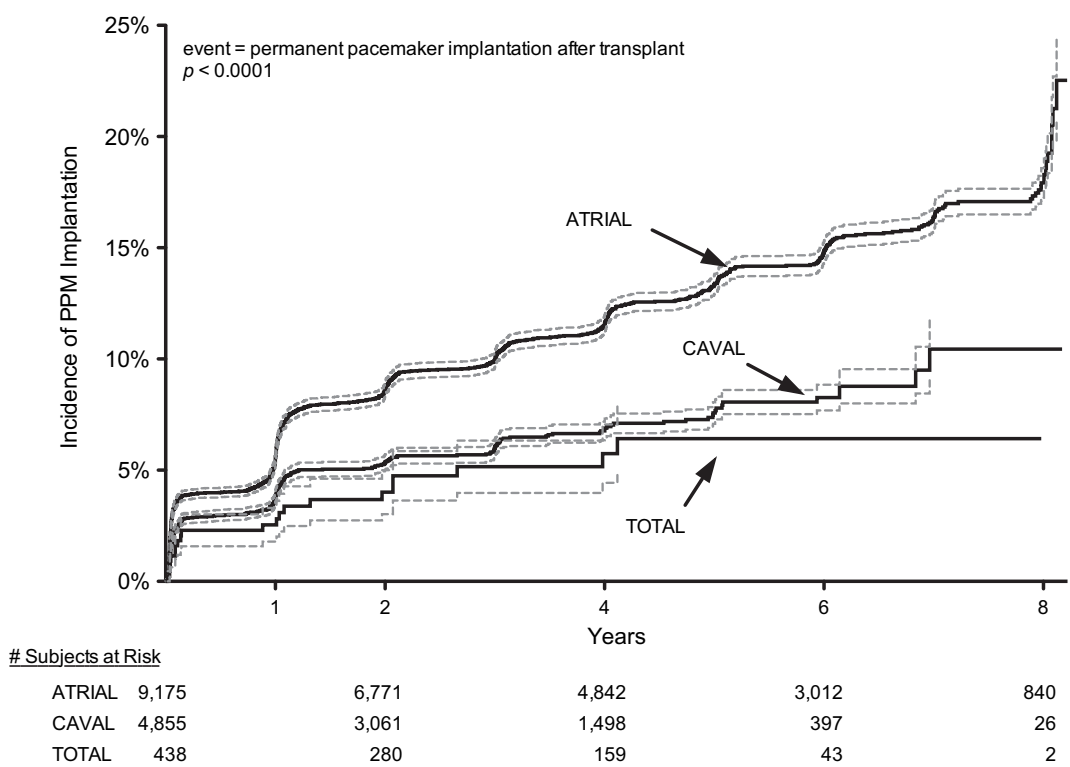

FIGURE 3. Kaplan-Meier estimates of the cumulative hazard of permanent pacemaker insertion after transplantation. Eight-year estimates are illustrated as a function of the type of transplant anastomosis: CAVAL, Bicaval anastomosis; ATRIAL, biatrial anastomosis; TOTAL, total heterotopic transplant anastomosis. Numbers of subjects at risk at each time point are given across the bottom, and standard errors are shown by the dashed lines. $P<.0001$ (caval vs atrial). 


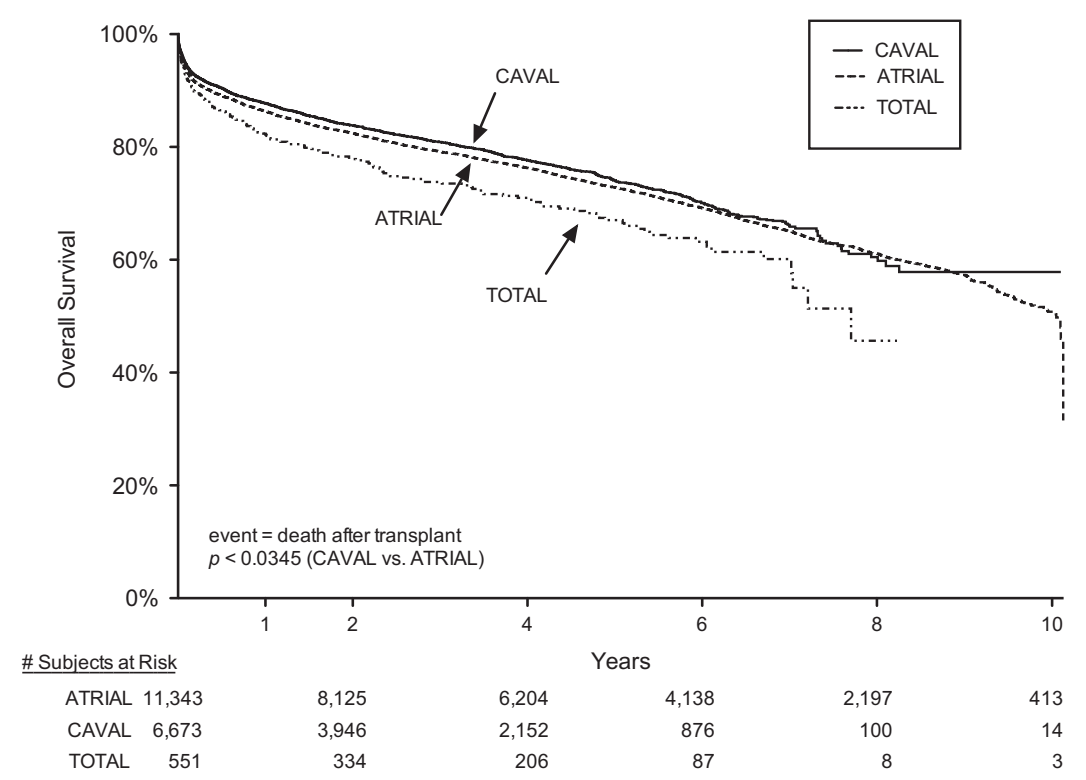

FIGURE 4. Kaplan-Meier estimates of cumulative long-term survival after transplantation. Ten-year estimates are illustrated as a function of the type of transplant anastomosis: CAVAL, bicaval anastomosis; ATRIAL, biatrial anastomosis; TOTAL, total heterotopic transplant anastomosis. Numbers of subjects at risk at each time point are given across the bottom. $P=.0345$ (caval vs atrial).

decreased atrioventricular valve regurgitation (especially the tricuspid valve).$^{11}$ In some studies these have resulted in demonstrable improvements in cardiac output and pulmonary vascular resistance 12 months after transplantation. ${ }^{11}$

The effect of differences in the need for posttransplantation PPM implantation on mortality is not clear. Consistent with previous studies, we have demonstrated a significantly higher rate of PPM implantation before discharge in patients with a biatrial anastomosis, with an odds ratio of 3.1 in multivariate analysis. ${ }^{8,10,27,28}$ Rothman and coworkers ${ }^{29}$ demonstrated increased levels of sinus node dysfunction (attributed to surgical damage) in patients with biatrial anastomoses. Our data, which show a persistently increased need for pacemaker implantation over long-term follow-up, suggest that ongoing interference with conductance pathways or nodal function occurs, possibly because of healing, scar formation, and ongoing inflammation in the region of the anastomosis. Other than implant technique, the most important predictors of the need for PPM insertion were related to donor factors, especially increasing donor age and donor history of hypertension, suggesting the importance of subclinical donor cardiac disease on subtle aspects of posttransplantation cardiac function.

Whether long-term electrical dysfunction and pacemaker use explain the increased mortality or whether the increased mortality with biatrial anastomosis can be explained by the alterations in atrial hemodynamics shown by others ${ }^{8}$ remains unclear, and the UNOS dataset simply does not contain the information required to address the causal relationship between transplant anastomosis and long-term mortality. Similar limitations prevent an accurate assessment of the potential causative factors in the higher mortality evident within the total orthotopic heart transplantation group. It is possible that technical factors result in a higher incidence of pulmonary venous stenosis or left atrial dysfunction in these patients. Given the relative rarity of the use of this technique, the poor outcomes might also reflect poor outcomes among a specific subset of surgeons, centers, or patients, but without additional data, we can only speculate.

Interestingly, and in contrast to many previously published models of posttransplantation mortality (although not Weiss and associates ${ }^{15}$ ), our multivariate model does not include year of transplantation as a predictor of longterm mortality. We speculate on 2 possible causes for this discrepancy. First, by limiting the analysis to the most recent 10 years, we might have excluded a significant amount of the improvement in transplantation outcomes. More intriguingly, it is possible that the increasing prevalence of bicaval anastomoses might be a causative factor in the decreasing mortality after transplantation in more recent years.

Transplant center volume was only minimally correlated with the percentage of procedures performed by using the bicaval anastomosis. Center volume and anastomosis technique might also be confounded because high-volume centers are more likely to perform complex reoperative transplantations, particularly in patients with congenital heart disease, in whom bicaval anastomosis might not be possible. Critiques of studies examining the relationship between hospital volume and surgical outcomes often focus on the fact that simply reporting volume-outcomes relationships is less instructive than identifying the causal factors underlying the relationship. We speculate that a portion of 
TABLE 4. Cox proportional hazards model of long-term survival after transplantation

\begin{tabular}{lcc}
\hline \multicolumn{1}{c}{ Risk factor } & Hazard ratio & $\mathbf{9 5 \%}$ CI \\
\hline Extracorporeal membrane oxygenation & 2.60 & $1.69-3.98$ \\
Prostaglandin E & 1.83 & $1.06-3.18$ \\
Mechanical ventilation & 1.75 & $1.50-2.03$ \\
Donor age 60-69 y & 1.69 & $1.29-2.21$ \\
Cause: congenital heart disease & 1.57 & $1.29-1.92$ \\
Creatinine clearance $<40$ mL/min & 1.57 & $1.40-1.77$ \\
Donor age 50-59 y & 1.53 & $1.39-1.77$ \\
Bilirubin $>2.0$ mg/dL & 1.36 & $1.24-1.49$ \\
Anastomosis: total & $\mathbf{1 . 3 1} *$ & $\mathbf{1 . 1 1}-\mathbf{1 . 5 6}$ \\
Cause: retransplantation & 1.29 & $1.10-1.53$ \\
Donor age 40-49 y & 1.29 & $1.19-1.40$ \\
Recipient: history of diabetes & 1.29 & $1.20-1.38$ \\
Recipient: history of chronic & 1.28 & $1.10-1.48$ \\
obstructive pulmonary disease & & \\
Ischemic time $>4$ h & 1.21 & $1.12-1.31$ \\
Ventricular assist device & 1.19 & $1.10-1.30$ \\
Donor age 30-39 y & 1.19 & $1.09-1.29$ \\
Intravenous antibiotics within 2 wk & 1.16 & $1.06-1.28$ \\
Recipient: history of previous stroke & 1.16 & $1.03-1.30$ \\
Cause: restrictive cardiomyopathy & 1.14 & $0.90-1.45$ \\
Anastomosis: biatrial & $\mathbf{1 . 1 1} *$ & $\mathbf{1 . 0 4 - 1 . 1 9}$ \\
Age (risk per additional year) & 1.004 & $1.001-1.007$ \\
Cause: ischemic cardiomyopathy & 0.97 & $0.87-1.08$ \\
Cause: valvular cardiomyopathy & 0.95 & $0.7-1.14$ \\
Cause: hypertrophic cardiomyopathy & 0.92 & $0.69-1.22$ \\
Donor-recipient sex match & 0.92 & $0.86-0.98$ \\
Sex (male) & 0.91 & $0.85-0.98$ \\
\hline
\end{tabular}

*Compared with the bicaval group.

the improved outcomes at high-volume centers might be due to their use of the bicaval technique.

Examination of surgeon rather than center volume might be instructive in attempting to identify barriers to adoption of the bicaval technique, but these data are not available in UNOS. The duration of cardiopulmonary bypass and warm organ ischemia might also be instructive because the additional anastomosis has been criticized as taking longer. However, in our experience we believe the bicaval technique can be performed as quickly as, if not more quickly than, the biatrial technique. Even if concerns regarding operative time were accurate, our data clearly demonstrate a long-term survival advantage to the bicaval anastomosis.

\section{Limitations}

As described above, the UNOS dataset contains several limitations that hinder our analysis. Although we have used an established technique to deal with missing data, missing information still limits the power of our analysis. In particular, reoperative sternotomy is poorly entered, despite the possibility that it is an important factor in early posttransplantation mortality. More importantly in the context of this analysis of surgical techniques, no data in the
UNOS dataset address long-term atrial hemodynamic function or electrophysiology, which might better elucidate causal relationships between the type of anastomosis and long-term outcomes.

\section{CONCLUSIONS}

Despite these limitations, we can conclude that in a multivariate analysis controlling for a large number of other risk factors, the use of a bicaval anastomosis is associated with improved long-term survival and freedom from PPM implantation. In addition, short-term benefits, including a decreased length of stay (likely related to reductions in the need for PPM before discharge), have also been clearly demonstrated. Accordingly, we recommend the use of the bicaval anastomosis for cardiac transplantation, except where anatomic challenges preclude its use.

We thank UNOS for supplying these data and Katarina Anderson for her assistance with our analysis.

\section{References}

1. Barnard CN. The operation. A human cardiac transplant: an interim report of a successful operation performed at Groote Schuur Hospital, Cape Town. S Afr Med J. 1967;41:1271-4.

2. Lower RR, Shumway NE. Studies on orthotopic homotransplantation of the canine heart. Surg Forum. 1960;11:18-9.

3. Leyh RG, Jahnke AW, Kraatz EG, Sievers HH. Cardiovascular dynamics and dimensions after bicaval and standard cardiac transplantation. Ann Thorac Surg. 1995;59:1495-500.

4. Freimark D, Silverman JM, Aleksic I, et al. Atrial emptying with orthotopic heart transplantation using bicaval and pulmonary venous anastomoses: a magnetic resonance imaging study. J Am Coll Cardiol. 1995;25:932-6.

5. Banner NR, Khaghani A, Fitzgerald M, Mitchell AG. The expanding role of cardiac transplantation. In: Unger F, ed. Assisted circulation. Berlin, Germany: Springer-Verlag; 1989.

6. Sievers HH, Weyand M, Kraatz EG, Bernhard A. An alternative technique for orthotopic cardiac transplantation, with preservation of the normal anatomy of the right atrium. Thorac Cardiovasc Surg. 1991;39:70-2.

7. Sarsam MA, Campbell CS, Yonan NA, Deiraniya AK, Rahman AN. An alternative surgical technique in orthotopic cardiac transplantation. J Card Surg. 1993;8: 344-9.

8. Schnoor M, Schäfer T, Lühmann D, Sievers HH. Bicaval versus standard technique in orthotopic heart transplantation: a systematic review and meta-analysis. J Thorac Cardiovasc Surg. 2007;134:1322-31.

9. Morgan JA, Edwards NM. Orthotopic cardiac transplantation: comparison of outcome using biatrial, bicaval, and total techniques. J Card Surg. 2005;20:102-6.

10. Meyer SR, Modry DL, Bainey K, et al. Declining need for permanent pacemaker insertion with the bicaval technique of orthotopic heart transplantation. Can J Cardiol. 2005;21:159-63.

11. Grande AM, Rinaldi M, D'Armini AM, et al. Orthotopic heart transplantation standard versus bicaval technique. Am J Cardiol. 2000;85:1329-33.

12. Aziz TM, Burgess MI, El-Gamel A, et al. Orthotopic cardiac transplantation technique: a survey of current practice. Ann Thorac Surg. 1999;68:1242-6.

13. Aziz T, Burgess M, Khafagy R, et al. Bicaval and standard techniques in orthotopic heart transplantation: medium-term experience in cardiac performance and survival. J Thorac Cardiovasc Surg. 1999;118:115-22.

14. el-Gamel A, Deiraniya AK, Rahman AN, Campbell CS, Yonan NA. Orthotopic heart transplantation hemodynamics: does atrial preservation improve cardiac output after transplantation? J Heart Lung Transplant. 1996;15:564-71.

15. Weiss ES, Nwakanma LU, Russell SB, Conte JV, Shah AS. Outcomes in bicaval versus biatrial techniques in heart transplantation: an analysis of the UNOS database. J Heart Lung Transplant. 2008;27:178-83.

16. Rubin DB. Multiple imputation for nonresponse in surveys. New York: Wiley; 1987. 
17. Ambler G, Omar RZ, Royston P. A comparison of imputation techniques for handling missing predictor values in a risk model with a binary outcome. Stat Methods Med Res. 2007;16:277-98.

18. Newgard CD. The validity of using multiple imputation for missing outof-hospital data in a state trauma registry. Acad Emerg Med. 2006;13: 314-24.

19. Shrive FM, Stuart H, Quan H, Ghali WA. Dealing with missing data in a multiquestion depression scale: a comparison of imputation methods. BMC Med Res Methodol. 2006;6:57.

20. Blanche C, Valenza M, Aleksic I, Czer LS, Trento A. Technical considerations of a new technique for orthotopic heart transplantation. Total excision of recipient's atria with bicaval and pulmonary venous anastomoses. J Cardiovasc Surg. 1994; 35:283-7.

21. Blanche C, Nessim S, Quartel A, et al. Heart transplantation with bicaval and pulmonary venous anastomoses. A hemodynamic analysis of the first 117 patients. J Cardiovasc Surg. 1997;38:561-6.

22. Sun JP, Niu J, Banbury MK, et al. Influence of different implantation techniques on long- term survival after orthotopic heart transplantation: an echocardiographic study. J Heart Lung Transplant. 2007;26:1243-8.
23. Omar RZ, Ambler G, Royston P, Eliahoo J, Taylor KM. Cardiac surgery risk modeling for mortality: a review of current practice and suggestions for improvement. Ann Thorac Surg. 2004;77:2232-7.

24. Newgard CD. The validity of using multiple imputation for missing out-ofhospital data in a state trauma registry. Acad Emerg Med. 2006;13:314-24.

25. Shrive FM, Stuart H, Quan H, Ghali WA. Dealing with missing data in a multiquestion depression scale: a comparison of imputation methods. BMC Med Res Methodol. 2006;6:57.

26. Freimark D, Czer LS, Aleksic I, et al. Improved left atrial transport and function with orthotopic heart transplantation by bicaval and pulmonary venous anastomoses. Am Heart J. 1995;130:121-6.

27. Aleksic I, Freimark D, Blanche C, et al. Resting hemodynamics after total versus standard orthotopic heart transplantation in patients with high preoperative pulmonary vascular resistance. Eur J Cardiothorac Surg. 1997; 11:1037-44.

28. Blanche C, Nessim S, Quartel A, et al. Heart transplantation with bicaval and pulmonary venous anastomoses. A hemodynamic analysis of the first 117 patients. J Cardiovasc Surg (Torino). 1997;38:561-6.

29. Rothman SA, Jeevanandam V, Combs WG, et al. Eliminating bradyarrhythmias after orthotopic heart transplantation. Circulation. 1996;94:II278-82. 


\section{MATERIALS AND METHODS}

This section details both the importance of the methods used to handle missing data in analysis of the UNOS dataset and the specific methods used in our analysis. The UNOS database contains significant amounts of missing data (Table E1)

\section{An Overview of the Problem of Missing Data}

What follows is a brief discussion of the statistical challenges raised by the presence of missing values in research datasets. For additional information, a range of publications with varying levels of statistical complexity is available. ${ }^{\text {E1-E7 }}$ Various methods of handling missing data have been described. Commonly used methods include complete case analysis (or listwise deletion), available case analysis, and overall mean imputation. ${ }^{\mathrm{E} 3}$ Although these have the advantage of being relatively simple to implement and easy to understand, they often result in a reduction in sample size and resultant loss of power; more importantly, they also commonly lead to severely biased estimates of statistical associations. ${ }^{\mathrm{E} 2, \mathrm{E} 3}$

Imputation is simply the replacement of missing values by using estimates of those values. Mean imputation is a commonly used method in which the mean of the nonmissing values is substituted for the missing values. Unfortunately, this method assumes that the missing observations are missing completely at random (ie, the probability of a particular observation being missing is unrelated to the value of the missing observation or any other variable in the dataset). This method will result in severely biased estimates when this unlikely assumption is not met and might result in biased estimates, even when the assumption is met. ${ }^{\mathrm{E} 3}$

A better method of imputing the missing values is to estimate them based on a multivariate analysis of the known variables. These estimates are then substituted for the missing variables in subsequent analyses. The drawback of this method is that subsequent analyses are performed as if those substituted values were observed rather than simply estimated, which results in overestimation of the precision of the associations (ie, underestimation of standard errors, $P$ values, and $95 \%$ confidence intervals). ${ }^{\mathrm{E} 2, \mathrm{E} 3}$

Multiple imputation attempts to solve the problem of overestimation of precision by creating multiple datasets, each with imputations drawn randomly from the estimated distribution of the missing values. Each of the multiple datasets is then analyzed, and statistical associations are estimated along with measures of precision. The estimates are then averaged across the datasets, and the measures of precision are combined. The result is an estimate of the statistical association and an estimate of the precision of that association that accurately reflect the error introduced by substituting estimated values for missing values. ${ }^{\mathrm{E} 1, \mathrm{E} 3}$

\section{Importance of Missing Data in the UNOS Dataset}

Although Weiss and associates ${ }^{\mathrm{E} 8}$ did not describe specific statistical analysis techniques or report the frequency of missing data, an analysis of the UNOS database from 1995 to 2005 suggests that of the 16,962 patients undergoing transplantations over that time period, only $10,799(63.7 \%)$ had complete information in the variables included in their multivariate model and were therefore eligible for inclusion in the model. This much smaller sample size might explain the lack of statistical power; in addition, given the likelihood of introducing bias into statistical analyses by using complete case analysis (particularly where the percentage of missing data is high), ${ }^{\mathrm{E} 3}$ the results of an analysis in which more than $35 \%$ of samples must be excluded should be viewed with skepticism. Multiple imputation has been used by other authors to develop accurate statistical estimates from the UNOS dataset despite the rate of missing data. ${ }^{\text {E9-E11 }}$

\section{Handling of Missing Data in This Study}

Missing variables were imputed by using the MI procedure of SAS 9.13 for Windows. Because of limitations of computing power, not all variables could be included in the imputation step; variables included are listed in Table E2. The MI procedure uses a Markov chain Monte Carlo method in which the missing values are replaced by $m(m=10$ in this study) simulated versions. Because simulation studies suggest that it provides the best estimates in situations in which the proportion of missing values exceeds $30 \%$, full-data imputation (IMPUTE $=$ FULL) was used. ${ }^{\mathrm{E} 4}$ The maximum likelihood estimates for the means and covariance matrix of a multivariate normal distribution of the dataset with missing values was computed by using the expectation-maximization algorithm with 400 maximal iterations. Odds/hazard ratios and $95 \%$ confidence intervals were estimated by using the MIANALYZE procedure.

\section{References}

E1. Rubin DB. Multiple imputation for nonresponse in surveys. New York: Wiley; 1987.

E2. Greenland S, Finkle WD. A critical look at methods for handling missing covariates in epidemiologic regression analyses. Am J Epidemiol. 1995;142:1255-64.

E3. Donders AR, van der Heijden GJ, Stijnen T, Moons KG. Review: a gentle introduction to imputation of missing values. J Clin Epidemiol. 2006;59:1087-91.

E4. Canchola A, Neilands B, Catania A. Imputation strategies for sexual orientation using SAS PROC MI. In: the Proceedings of the Tenth Annual Western Users of SAS Software Conference, San Diego. 2002;6

E5. Barnard J, Meng XL. Applications of multiple imputation in medical studies: from AIDS to NHANES. Stat Methods Med Res. 1999;8:17-36.

E6. Little RJA. Regression with missing X's: a review. J Am Stat Assoc. 1992;87: 1227-37.

E7. Vach W. Logistic regression with missing values in the covariates. New York: Springer-Verlag; 1994.

E8. Weiss ES, Nwakanma LU, Russell SB, Conte JV, Shah AS. Outcomes in bicaval versus biatrial techniques in heart transplantation: an analysis of the UNOS database. J Heart Lung Transplant. 2008;27:178-83.

E9. Gasink LB, Blumberg EA, Localio AR, Desai SS, Israni AK, Lautenbach E. Hepatitis $\mathrm{C}$ virus seropositivity in organ donors and survival in heart transplant recipients. JAMA. 2006;296:1843-50.

E10. Lederer DJ, Benn EK, Barr RG, et al. Racial differences in waiting list outcomes in chronic obstructive pulmonary disease. Am J Respir Crit Care Med. 2008; $177: 450-4$

E11. Thabut G, Ravaud P, Christie JD, et al. Determinants of the survival benefit of lung transplantation in patients with chronic obstructive pulmonary disease. Am J Respir Crit Care Med. 2008;177:1156-63. 
TABLE E1. Frequency of missing data for all variables included in multivariate models predicting both long-term and short-term risk of mortality $(n=20,069)$ *

\begin{tabular}{|c|c|}
\hline Risk factor & $\begin{array}{c}\text { Percentage } \\
\text { missing }\end{array}$ \\
\hline Albumin level at listing & 43.5 \\
\hline Previous sternotomy & 35.2 \\
\hline Panel reactive antibody level & 35.0 \\
\hline Donor on T3 at organ retrieval & 30.3 \\
\hline History of hepatitis B & 12.0 \\
\hline Organ ischemic time & 9.3 \\
\hline Transfusion between listing and transplantation & 9.2 \\
\hline Bilirubin level at transplantation & 7.8 \\
\hline Donor on T4 at organ retrieval & 7.7 \\
\hline History of COPD & 7.3 \\
\hline Creatinine clearance at transplantation & 5.6 \\
\hline BMI at transplantation & 5.6 \\
\hline Dialysis at transplantation & 4.2 \\
\hline Recipient/donor weight ratio & 3.6 \\
\hline History of stroke & 3.0 \\
\hline History of hepatitis $\mathrm{C}$ & 2.2 \\
\hline Donor bilirubin & 2.2 \\
\hline History of diabetes & 1.9 \\
\hline Donor history of hypertension & 0.7 \\
\hline Donor cause of death & 0.2 \\
\hline Donor age & 0.1 \\
\hline Intravenous antibiotics within $2 \mathrm{wk}$ & 0.06 \\
\hline Hospital location (ICU vs non-ICU vs out of hospital) & 0.01 \\
\hline Status at transplantation & 0.01 \\
\hline Cause & 0.0 \\
\hline Sex & 0.0 \\
\hline Use of extracorporeal membrane oxygenation & 0.0 \\
\hline Use of ventricular assist device & 0.0 \\
\hline Use of intra-aortic balloon pump & 0.0 \\
\hline Mechanical ventilation & 0.0 \\
\hline Inhaled nitric oxide & 0.0 \\
\hline Prostaglandin E & 0.0 \\
\hline Donor ABO type & 0.0 \\
\hline Donor cigarette use & 0.0 \\
\hline Donor sex & 0.0 \\
\hline Donor on inotropes/pressors & 0.0 \\
\hline Recipient ABO type & 0.0 \\
\hline Transplantation year & 0.0 \\
\hline Anastomosis: total & $0.0 \dagger$ \\
\hline Anastomosis: biatrial & $0.0 \dagger$ \\
\hline \multicolumn{2}{|c|}{$\begin{array}{l}C O P D \text {, Chronic obstructive pulmonary disease; } B M I \text {, body mass index; } I C U \text {, intensive } \\
\text { care unit. *Variables with zero missing values are often variables calculated based on } \\
\text { responses in which no option was given for "no," and thus null results are assumed to } \\
\text { be "no" responses, resulting in no missing values. } \dagger \text { Because anastomosis type was the } \\
\text { focus of this analysis, missing values were not imputed for this variable, and } 327 \text { pa- } \\
\text { tients without anastomosis data were excluded. }\end{array}$} \\
\hline
\end{tabular}

TABLE E2. List of variables included in the multiple imputation procedure for imputation of missing variables

\begin{tabular}{ll}
\hline Albumin & $\begin{array}{c}\text { Location (ICU, non-ICU, } \\
\text { out of hospital) } \\
\text { Mechanical ventilation }\end{array}$ \\
$\begin{array}{l}\text { Body mass index } \\
\text { CMV status }\end{array}$ & $\begin{array}{l}\text { Peak PRA level } \\
\text { Previous sternotomy }\end{array}$ \\
$\begin{array}{l}\text { Distance between recovery and } \\
\text { transplant centers }\end{array}$ & Recipient bilirubin \\
Donor age & Recipient dialysis \\
Donor bilirubin & \\
Hepatitis B & Status at transplantation \\
Hepatitis C & Transplantation year \\
Ischemic time & Use of T3 \\
\hline
\end{tabular}

$I C U$, Intensive care unit; $P R A$, panel reactive antibody; $C M V$, cytomegalovirus. 